\title{
Staggering of angular momentum distribution in fission
}

\author{
Pierre Tamagno^ and Olivier Litaize \\ CEA, DEN, DER, SPRC, Cadarache, 13108 Saint-Paul-lez-Durance, France
}

\begin{abstract}
We review here the role of angular momentum distributions in the fission process. To do so the algorithm implemented in the FIFRELIN code [1] is detailed with special emphasis on the place of fission fragment angular momenta. The usual Rayleigh distribution used for angular momentum distribution is presented and the related model derivation is recalled. Arguments are given to justify why this distribution should not hold for low excitation energy of the fission fragments. An alternative ad hoc expression taking into account low-lying collectiveness is presented as has been implemented in the FIFRELIN code. Yet on observables currently provided by the code, no dramatic impact has been found. To quantify the magnitude of the impact of the low-lying staggering in the angular momentum distribution, a textbook case is considered for the decay of the ${ }^{144} \mathrm{Ba}$ nucleus with low excitation energy.
\end{abstract}

\section{Introduction}

The fission process is probably the most complex - and the less accurately understood - phenomenon of nuclear physics. This is mostly due to the fact that many degrees of freedom are involved in the process and that it is extremely difficult to experimentally separate contributions. For instance, kinetic energy of fission fragments are at-best measured with respect to mass number $A$ and fine isotope decompositions are rare. Various isotopes are generated in fission with various "initial" states, and additionally for each of them the decay process is also not perfectly known. As only average experimental quantities are available, it is important to have models that are consistent and as accurate as possible so that experimental data can be properly interpreted. As mentioned above, the decay process of a fission fragment is not perfectly known. Indeed this decay process depends on the nuclear property of the isotope, such as the level scheme and transition branching ratios (or decay widths). These quantities can be obtained experimentally with spectroscopy techniques but the number of accurately measurable levels is limited. The limitations are not only due to statistics or to experimental resolution but can also come from the unstable nature of the isotope ( $\beta$-decay). Therefore to describe the nuclear structure above some given level, models are used to fulfill gaps. In the present work we highlight the role of a specific degree of freedom in the fission phenomenon: the angular momenta of levels of fission fragments. To illustrate where these distributions can be used in fission models, we present first the model implemented in the FIFRELIN code [1] to describe fission observables. Then we present why we expect that current angular distribution models should be incorrect in the low excitation energy region. We detail then an ad hoc modification that we implemented in FIFRELIN

^e-mail: pierre.tamagno@cea.fr 
and that should be more accurate. Finally we present a simple case of a decay of a fission fragment with a low excitation energy so that the impact of our modification can be quantified.

\section{Fission modeling in FIFRELIN}

\subsection{Generation of initial fragments}

Particle emission in the FIFRELIN code is based on the Bečvár algorithm [2] for the simulation of $\gamma$ cascades with extension to the emission of prompt neutrons. The decay algorithm and fission fragment (FF) generation are stochastic. Fission events are sampled many times and decay properties of fragments are recorded so that average properties can be provided to compare with experimental data. More detailed quantities can also be provided as well as correlations between observables. The first step in a simulation consists in sampling FF and FF states. The mass fragmentation is sampled in an experimental yield $Y(A)$ such as shown in Fig. 1 for the ${ }^{252} \mathrm{Cf}(\mathrm{sf})$ reaction. The charge fragmentation is sampled according to a gaussian distribution $P_{Z}$ (see Fig. 1) with a mean value $Z_{\mathrm{p}}$ that is given by the unchanged-charge-density value shifted by $\pm \Delta Z$. For instance, for the light fragment, the most-probable charge is given by $Z_{\mathrm{L}, \mathrm{p}}=\left(Z_{\mathrm{CN}} / A_{\mathrm{CN}}\right) A_{\mathrm{L}}+\Delta Z\left(A_{\mathrm{L}}\right)$, where the polarization $\Delta Z$ is also obtained from experimental data as illustrated in Fig. 1. Once both fragments are defined, the kinetic energy of the light fragment is sampled from experimental kinetic energy data (mean values $\langle K E\rangle(A)$ and standard deviations $\left.\sigma_{K E}(A)\right)$ and the kinetic energy of the heavy fragment is deduced from momentum conservation.
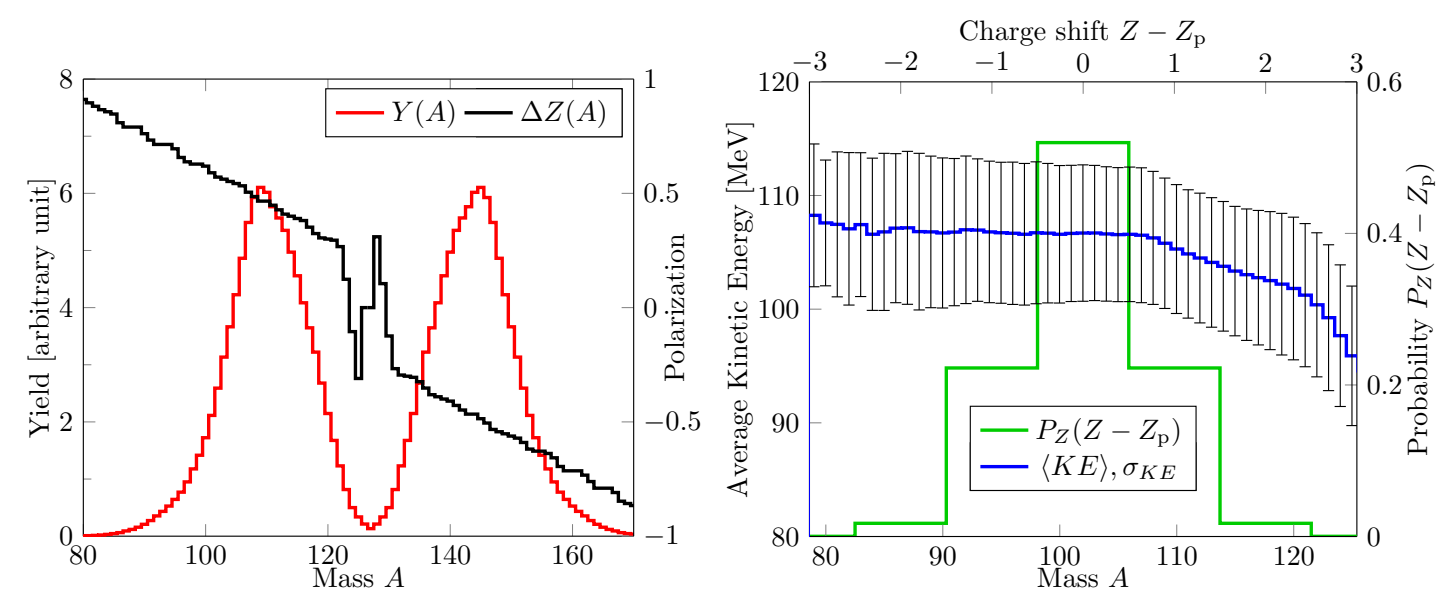

Figure 1. Scission fission yield $Y(A)$ and charge polarization $\Delta Z$ for the ${ }^{252} \mathrm{Cf}(\mathrm{sf})$ reaction (left). Related fragments average kinetic energy $\langle K E\rangle(A)$ and standard deviation $\sigma_{K E}(A)$ (right).

From the defined fragments properties, the total excitation energy $T X E$ can be calculated from the total released energy $Q$ by $T X E=Q-K E\left(A_{\mathrm{H}}\right)-K E\left(A_{\mathrm{L}}\right)$. The total intrinsic excitation energy is given by $E^{*}=T X E-E_{\mathrm{L}}^{\text {rot }}-E_{\mathrm{H}}^{\text {rot }}+B_{\mathrm{n}}+E_{\mathrm{n}}^{\text {kin }}$, where $E_{\mathrm{L}}^{\text {rot }}$ and $E_{\mathrm{H}}^{\text {rot }}$ are respectively the rotational energies of the light and heavy fragments. These rotational energies are obtained by: $E^{\text {rot }}=J(J+1) / 2 k I$ where $I=\frac{2}{5} M R^{2}\left(1+0.31 \beta_{2}\right)$ is the rigid-body moment of inertia of the fragment and $k$ is an adjustable parameter. To determine the rotational energy of the fragment, angular momentum $J$ is required. It is sampled from a Rayleigh distribution:

$$
P(J)=\frac{J+1 / 2}{\sigma^{2}} \exp \left[-\frac{(J+1 / 2)^{2}}{2 \sigma^{2}}\right],
$$


where $\sigma^{2}$ is the so-called spin cutoff parameter. In practice there is one adjustable spin cutoff parameter for each group of fragments $\left(\sigma_{\mathrm{H}}\right.$ and $\left.\sigma_{\mathrm{L}}\right)$. They are only used in the initial fragmentation sampling. Finally, the initial parity of both fragments is uniformly sampled and the total intrinsic excitation $E^{*}$ is dispatched between fragments according to a temperature ratio law [1] involving two additional parameters $R_{\mathrm{T}}^{\min }$ and $R_{\mathrm{T}}^{\max }$. We emphasize here that only 5 parameters are adjusted in practice to reproduce average quantities such as prompt neutron multiplicities for the heavy and light fragments $\left(\bar{v}_{\mathrm{H}}\right.$ and $\bar{v}_{\mathrm{L}}$ respectively).

\subsection{Fragment decay}

At the end of the fragmentation sampling, one has two fragments with well-defined initial states. For instance, for the heavy fragment $\left(A_{\mathrm{H}}, Z_{\mathrm{H}}, E_{\mathrm{H}}^{*}, J_{\mathrm{H}}, \pi_{\mathrm{H}}\right)$. The decay cascade is simulated in a similar manner for both fragments, therefore we only consider an arbitrary fragment state $\left(A, Z, E^{*}, J, \pi\right)$. The initial state of the fragment is usually at higher energy than the highest known level. Therefore its decay from the initial state down to a long-lived state requires modeling of unknown levels and transitions. This energy region is illustrated in Fig. 2. Below the highest known level $\left(E_{\text {cut }}\right)$ all transitions are experimentally known [3]. Above $E_{\text {cut }}$ some levels can be known but additional levels must be sampled to complete the level scheme. Levels are added until a maximum number of levels (or a maximum level density) criteria is matched, corresponding to an energy $E_{\text {bin }}$. Above $E_{\text {bin }}$ the decay is simulated with equal-width energy bins. The probability of emitting a particle $p$ with properties $\alpha$ from state $i$ to state $f$ is given by the Hauser-Feshbach statistical model:

$$
P_{p}(i \rightarrow f, \alpha)=\frac{\Gamma_{p}(i \rightarrow f, \alpha)}{\sum_{f^{\prime}, p^{\prime}, \alpha^{\prime}} \Gamma_{p^{\prime}}\left(i \rightarrow f^{\prime}, \alpha^{\prime}\right)} .
$$

In Eq. (2), $\Gamma$ are the level decay widths. For gamma emission with multipolarity $X L$, the level decay width is given by

$$
\Gamma_{\gamma}(i \rightarrow f, X L)=\left(E_{i}-E_{f}\right)^{2 L+1} \frac{S_{\gamma}^{X L}\left(E_{i}-E_{f}\right)}{\rho\left(E_{i}, J_{i}, \pi_{i}\right)} .
$$

When the excitation energy is higher than the neutron separation energy $S_{\mathrm{n}}$, the fragment can emit a neutron. Therefore additional decay channels must be considered. The related decay widths are obtained from optical model calculation with inverse kinematics, yielding

$$
\Gamma_{\mathrm{n}}(i \rightarrow f, \ell j)=\frac{T_{\ell j}\left(E_{\mathrm{n}}^{\mathrm{inv}}\right)}{\rho\left(E_{i}, J_{i}, \pi_{i}\right)} .
$$

Examples of gamma strength functions $S_{\gamma}^{X L}$ and neutron transmission coefficients $T_{\ell_{j}}$ can be seen in Fig. 3. In conclusion one can notice from Eqs. (3) and (4) and from the unknown levels sampling that the spin distribution $P(J)$ (that is hidden in $\rho$ in Eqs. (3) and (4)) plays an important role in different places in the decay simulation.

\section{Angular momentum distribution}

\subsection{Rayleigh distribution}

Before getting interested in liabilities of Eq. (1), we briefly recall here how it can be obtained [4]. Considering a large number of nucleons (Fermi gas) having their spin projection $m_{i}$ randomly distributed. The total spin projection $M=\sum_{i} m_{i}$ follows a Gaussian distribution (central limit theorem) 

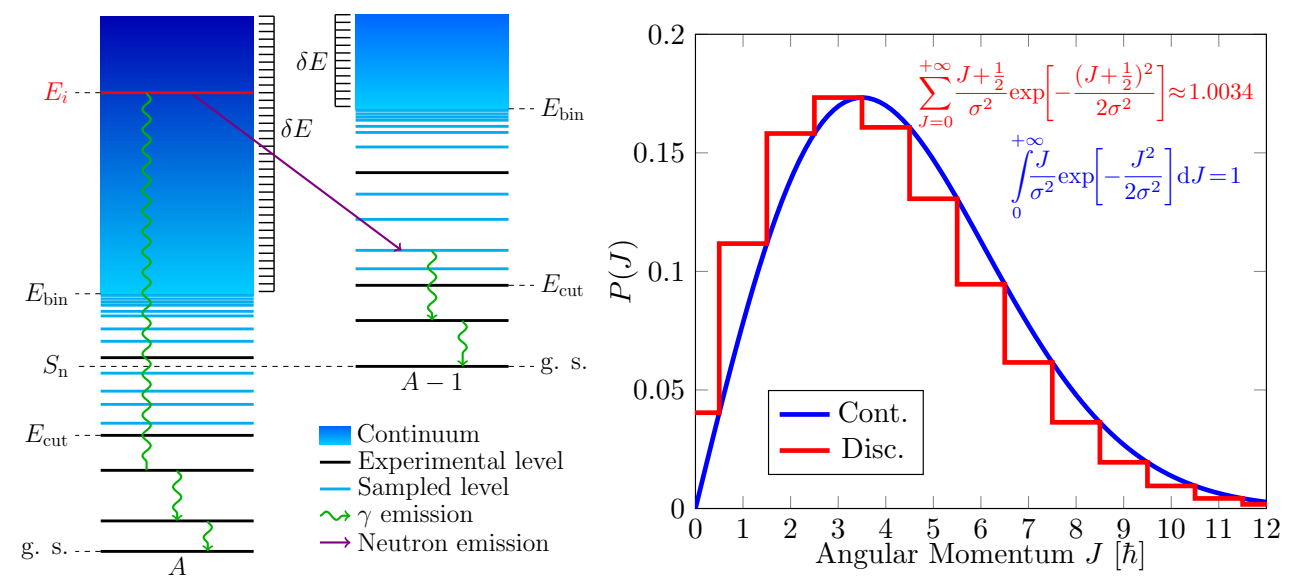

Figure 2. Schematic decay cascade simulated in FIFRELIN (left). Angular momentum distributions obtained with both types shown in Eq. (5) with a spin cutoff $\sigma=3.5 \hbar$ (right).

i.e. $P(M) \propto \exp \left(-M^{2} / 2 \sigma^{2}\right)$. The probability that the system has a total angular momentum $J$ is then given by $P(J)=P(M=J)-P(M=J+1) \approx(J+1 / 2) / \sigma^{2} \exp \left[-(J+1 / 2)^{2} / 2 \sigma^{2}\right]$, which is Eq. (1). One can notice that Eq. (1) - although widely used - is not exactly a distribution. Indeed a Rayleigh distribution operates on a continuous variable, whereas Eq. (1) deals with discrete values of $J$. As a consequence, Eq. (1) is not normalized to unity:

$$
\sum_{J=0}^{+\infty} \frac{J+1 / 2}{\sigma^{2}} \exp \left[-\frac{(J+1 / 2)^{2}}{2 \sigma^{2}}\right] \neq 1 \quad \text { whereas } \quad \int_{0}^{+\infty} \frac{J}{\sigma^{2}} \exp \left[-\frac{J^{2}}{2 \sigma^{2}}\right] \mathrm{d} J=1 .
$$

One can also notice that Eq. (1) is used indifferently for even-even or for other nuclei. An illustration of both distributions of Eq. (5) is shown in Fig. 2. Beside the normalization issue, the discrete distribution should yield more low angular momenta (red curve is shifted). This is due to the $J+1 / 2$ term in the discrete expression [5], otherwise the occurrence probability of $J=0$ would be zero.
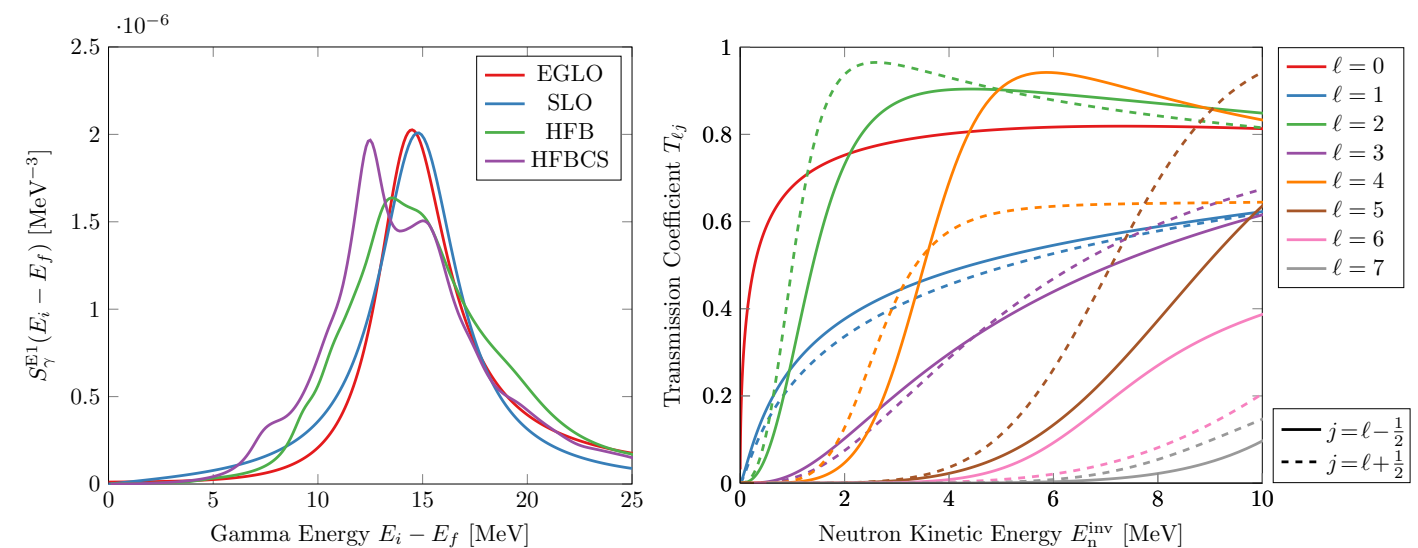

Figure 3. Examples of gamma strength functions (left) and neutron transmission coefficients for ${ }^{144} \mathrm{Ba}$ (right). 


\subsection{Collective footprint in level density}

This normalization and discrete-continuum difference are not the only liabilities of Eq. (1). Indeed one can notice discrepancies between expected results from low-lying levels analysis and the statistical model described in Sect. 3.1. A typical low-lying level scheme for ${ }^{154} \mathrm{Gd}$ is shown in Fig. 4. One can notice the well-known collective structures related to rotational bands built on the ground-state and on $\beta, \gamma$ or octupole vibrational states. The $K=0$ bands have a selective effect on the related band levels. For $K=0^{+}$only even value of $J$ are built, whereas for $K=0^{-}$only odd values of $J$ are present in the band. Additionally, no $0^{-}$states are present in these low-lying levels. From this example one can expect parity-driven structures in the angular momentum distribution. This collective states have a well-known impact on the total level density $\rho\left(E^{*}\right)\left(K_{\text {coll }}\right.$ and $K_{\text {vib }}$ enhancement factors [3]). This strong change in behavior between a Fermi gas and a collectiveness-driven system can also explain large differences than can be observed between Fermi-gas spin cutoff $\sigma_{\mathrm{F}}$ and "discrete" spin cutoff $\sigma_{\mathrm{d}}$ extracted from low-lying levels analysis [3].

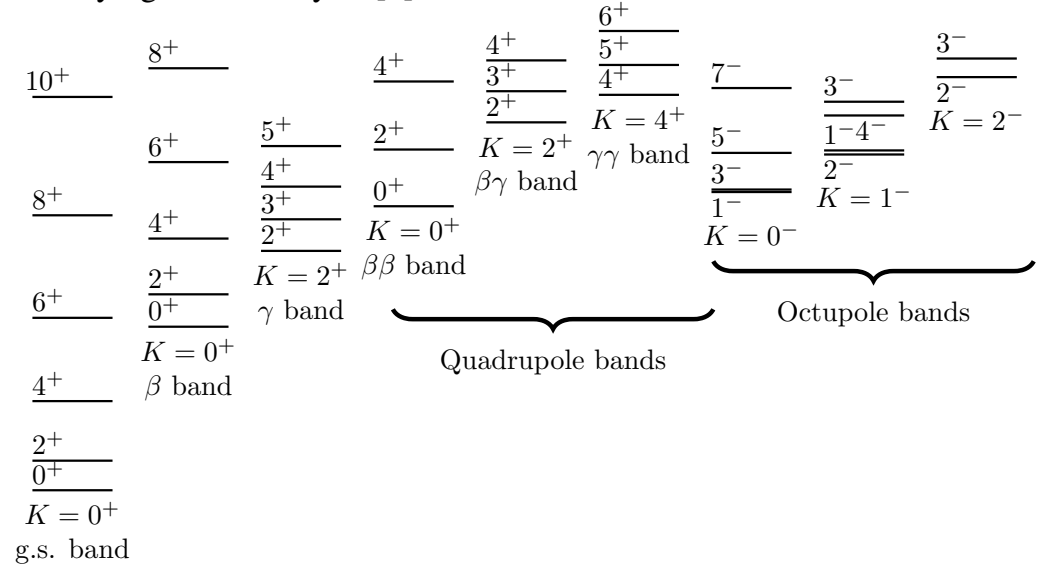

Figure 4. Low-lying collective bands in ${ }^{154} \mathrm{Gd}$ data taken from ENSDF [6].

An additional evidence of the presence of parity-driven enhancements can be obtained from Hartree-Fock-Bogoliubov calculations [7]. In Fig. 5 one can see an example of angular momentum distribution of ${ }^{144} \mathrm{Ba}$ obtained from combinatorial level density based on HFB theory. It can be easily seen that for low energies the staggering effect discussed above enhances even spins for even parity and odd spins for odd parity.
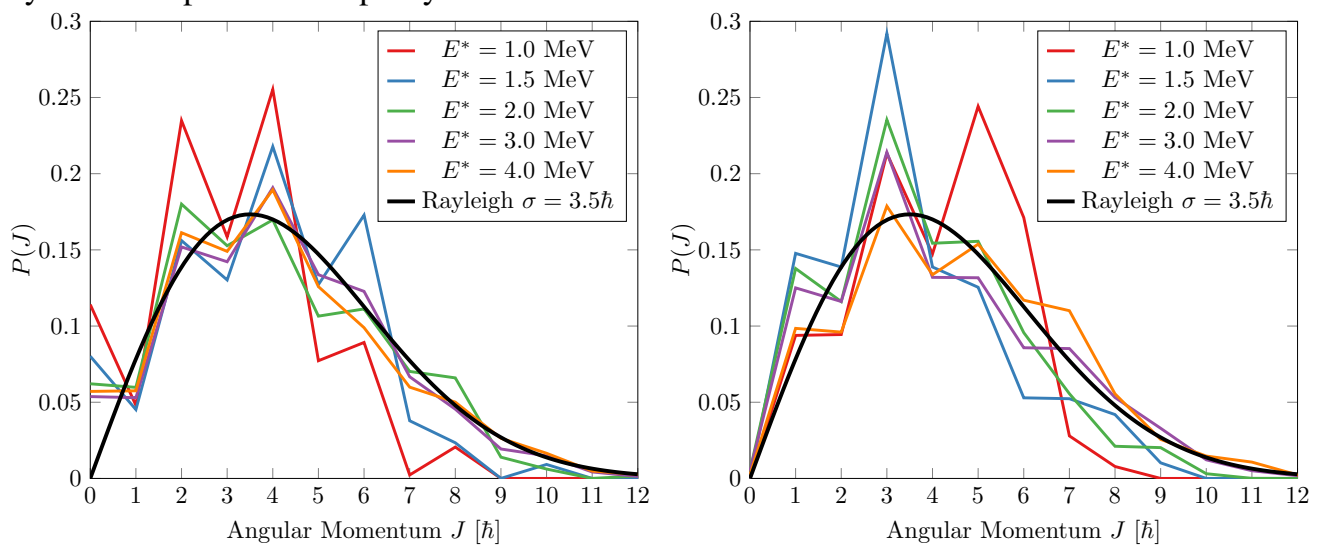

Figure 5. Angular momentum distributions obtained from HFB level density analysis for ${ }^{144} \mathrm{Ba}$, for positiveparity levels (left) and negative-parity levels (right). 


\section{Results on textbook case}

We investigated the impact of the staggering presented in Sect. 3.2 on fission observables. The usual Eq. (1) has thus been modified in FIFRELIN so that even-odd enhancements can be reproduced. We also had to modify the code so that parity is sampled before angular momentum so that different distributions can be used according to parity. The alternative parity-dependent angular momentum distribution used is:

$$
\tilde{P}(J)=\left[\frac{a(1-a)}{2}+1\right] P(J)+\frac{a(a+1)}{4}[P(J+1)+P(J-1)] \quad \begin{array}{ll}
a(\pi=+)=f \\
a(\pi=-)=-f
\end{array}
$$

In Eq. (6), $P(J)$ stands for the usual Rayleigh expression (Eq. (1)). Only even-even nuclei are impacted by this modification as it is expected that single-particle excitations quickly wash out the collective structures. In Eq. (6), $f$ is a parameter controlling the strength of the enhancement. For $f=0$ one obtains $\tilde{P}(J)=P(J)$, whereas for $f=1$ one only obtains selected spins. In Fig. 6, Eq. (6) is illustrated for different values of $f$. To take into account that the staggering disappear with increasing excitation energy, we introduced the energy dependency $f=\exp \left(-E^{*} / 1.8\right)$.

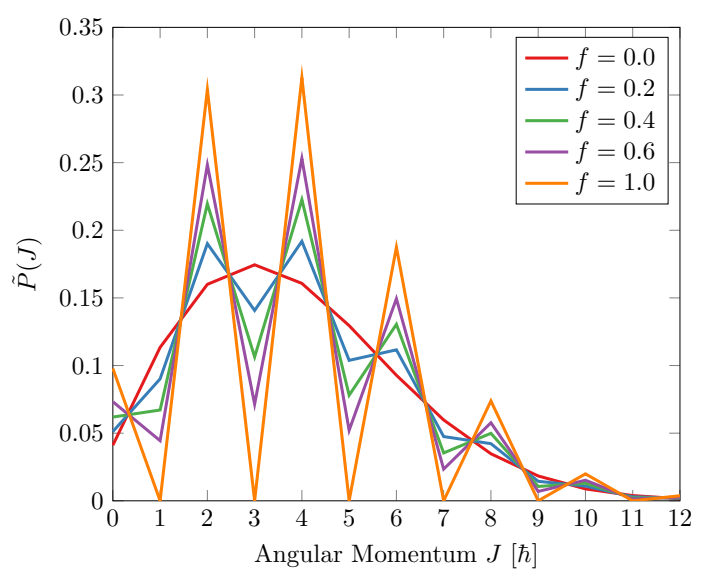

Figure 6. Ad hoc angular momentum distributions used to enhance even angular momenta for positive-parity states.

We expected that modifying the functional $P$ that is used in many places in the algorithm would have a significant impact on fission observables provided by the code, for instance gamma and neutron multiplicities and spectra or even isomeric ratios. Yet no such dramatic effect has been found. Two reasons explain this limited impact:

- At high excitation energy, many levels are available as "arrival" states i.e. the level density $\rho\left(E_{f}\right)$ is still high. Therefore if a transition become prohibited to a given level, there will be nearby another level with the proper spin and parity. The decay will then occur with an almost identical probability because the typical energy scale for the variation in gamma strength-function or neutron transmission coefficient is much larger than the average level spacing (see Fig. 3).

- At low excitation energy, experimental transition intensities are known. As they drive the HauserFeshbach sampling, spin-parity of experimental levels have limited impact on the decay cascade.

As the code relies on Monte Carlo sampling, quantifying this impact would require outrageous computation time. Therefore we tried to quantify this impact on a simpler case. The decay of a ${ }^{144} \mathrm{Ba}$ isotope exited in a $6^{+}$state at $E^{*}=1.5 \mathrm{MeV}$ (see Fig. 7) has been simulated with both models $P$ 
and $\tilde{P}$. We retained only the first 6 experimentally-known states. To simulate the decay cascade the code samples additional levels between the highest experimentally-known state and the initial energy. The energy and parity samplings are made before spin attributions therefore they are identical in both cases. It can be notice from Fig. 7 that $\tilde{P}$ favor even spins for positive parity and odd spins for negative parity. The simulated gamma intensities can be seen in Fig. 7. One can see that additional rays are observed in the modified case but with intensities about three orders of magnitude smaller than for the main transitions. This explains why differences on main events are not seen on global fission observables.
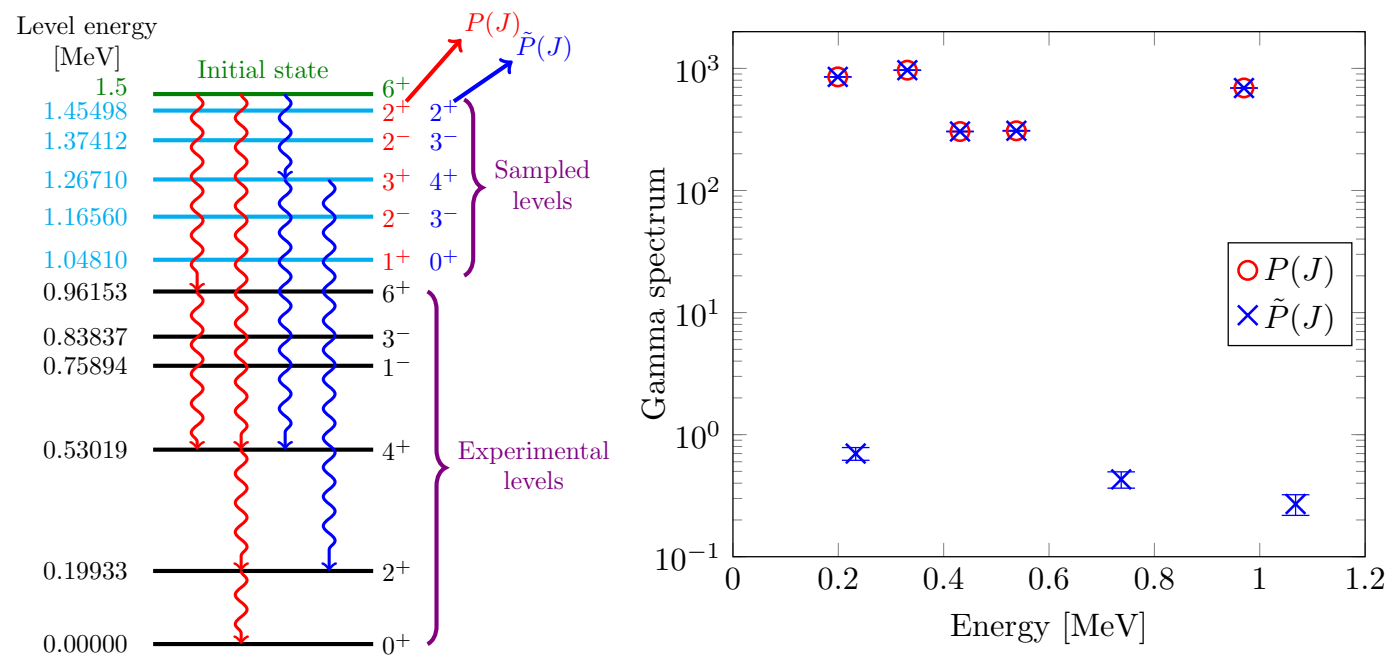

Figure 7. Level scheme obtained in the textbook decay of ${ }^{144} \mathrm{Ba}$ using both models for angular distribution (left). Corresponding calculated gamma intensities (right).

\section{Conclusion}

The usual Rayleigh distribution used for the description of angular momenta in nuclei is reviewed. It is shown that such a model is expected to be inaccurate at low excitation energies for even-even nuclei. An alternative expression is proposed to simulate the impact of collective states in the distribution. This modification has been shown to have limited impact on average fission observables and its impact has been quantified to be about three orders of magnitude smaller than main decay processes.

\section{References}

[1] O. Litaize, O. Serot, Phys. Rev. C 82, 054616 (2010)

[2] F. Bečvář, Nuclear Instruments and Methods in Physics Research A 417, 434 (1998)

[3] R. Capote, M. Herman, P. Obložinský, P.G. Young, S. Goriely, T. Belgya, A.V. Ignatyuk, A.J. Koning, S. Hilaire, V.A. Plujko et al., Nuclear Data Sheets 110, 3107 (2009)

[4] H.A. Bethe, Phys. Rev. 50, 332 (1936)

[5] A. Gilbert, A.G.W. Cameron, Canadian Journal of Physics 43, 1446 (1965)

[6] M.R. Bhat, in Nuclear Data for Science and Technology (1992), Evaluated Nuclear Structure Data File (ENSDF)

[7] S. Hilaire, personal communication 
